\title{
EEG Feature Classification Based on Grip Strength for $\mathrm{BCl}$ Applications
}

\section{Dong-Eun Kim, Je-Hun Yu, and Kwee-Bo Sim}

Department of Electrical and Electronics Engineering, Chung-Ang University, Seoul, Korea

\section{ljfis}

Received: Dec. 8, 2015

Revised : Dec. 24, 2015

Accepted: Dec. 24, 2015

Correspondence to: Kwee-Bo Sim

(kbsim@cau.ac.kr)

(CThe Korean Institute of Intelligent Systems

(c)This is an Open Access article distributed under the terms of the Creative Commons Attribution Non-Commercial License (http://creativecommons.org/licenses/ by-nc/3.0// which permits unrestricted noncommercial use, distribution, and reproduction in any medium, provided the original work is properly cited.

\begin{abstract}
Braincomputer interface (BCI) technology is making advances in the field of humancomputer interaction (HCI). To improve the BCI technology, we study the changes in the electroencephalogram (EEG) signals for six levels of grip strength: 10\%, 20\%, 40\%, 50\%, 70\%, and $80 \%$ of the maximum voluntary contraction (MVC). The measured EEG data are categorized into three classes: Weak, Medium, and Strong. Features are then extracted using power spectrum analysis and multiclass-common spatial pattern (multiclass-CSP). Feature datasets are classified using a support vector machine (SVM). The accuracy rate is higher for the Strong class than the other classes.
\end{abstract}

Keywords: Brain-computer interface, Electroencephalogram, Multi-common spatial pattern

\section{Introduction}

Human-computer interaction ( $\mathrm{HCI}$ ) is important thing for the communication with humans and devices. In the field of HCI, research related to braincomputer interfaces (BCIs), where devices are controlled using the electroencephalogram (EEG) signals of the brain, is being conducted for various applications. In this field, when a human is induced in a particular state, the extraction and application of his/her EEG features are necessary. Epileptic patients are characterized by recurring seizures, which results in abnormal EEG signals. A doctor can confirm a spike in the EEG, which is unlike a normal EEG, using BCI technology during a seizure or during a seizure-free period, and can therefore make an accurate diagnosis of the disease [1].

Similarly, application equipment such as wheelchairs can be controlled using the extracted EEG while considering the property of laterality in the brain when a human thinks or moves his/her body. Detection and feature extraction of the EEG signals are necessary for the application to a device based on the induced human state [2].

A previous study by the authors reported a correlation between the grip strength and the EEG signal of the general brain region, and analysis and classification of grip strength for artificial hand [3-5]. In this study, the participants gripped a strength measurement device at intervals of $10 \%$ of their maximum voluntary power. The previous study showed that the stronger the grip, the greater the power value in the three regions of the brain (frontal, central, and parietal). Following the previous research, this study investigates the relationship between the EEG signal and the strength for delicate motion control of an artificial arm of quadriplegic patients. In this experiment, the EEG of the participants was measured while they exerted their grip strength. The grip strength was divided into three classes: Weak, Medium, and Strong. In 
this study, the linear change in the $\beta$ and $\gamma$ waves was determined and then feature extraction and classification algorithms were applied.

\section{Background}

\subsection{Related Works}

The brain consists of four regions: the frontal lobe, parietal lobe, temporal lobe, and occipital lobe. EEG signals related to motor control are obtained from the motor cortex, which is located between the frontal lobe and the parietal lobe. Event-related desynchronization (ERD) / event-related synchronization (ERS) occurs when humans perform voluntary motor behavior. ERD decreases the amplitude of the EEG signal in $\alpha$ waves before voluntary motor behavior, and ERS increases the amplitude of the EEG signal in $\beta$ waves after voluntary motor behavior [6].

\subsection{Power Spectrum Analysis}

Power spectrum analysis translates time series data into frequency dimension, and therefore, this algorithm is useful for bio-signal analysis. This study analyzed $\beta$ and $\gamma$ waves to identify the voluntary behavior related to ERS.

\subsection{Multiclass-Common Spatial Pattern}

The CSP algorithm is used for feature extraction. Although CSP initially used only two classes, there is research on using multiple classes. This study applied a multiclass-CSP algorithm reported by Tang et al. [7]. CSP is a method that maximizes the variance of the selected class and the variance of the other non-selected classes is minimized so that the features of the EEG signal can be obtained. This process can be presented [7, 8] as follows:

$$
w=\underset{w \in \mathbb{R}^{N}}{\arg \max }\left\{\frac{w^{\mathrm{T}} R_{x \mid \mathrm{c}} w}{w^{\mathrm{T}} R_{x \mid \mathrm{c}^{\prime}} w}\right\}
$$

where $R_{x \mid \mathrm{c}}, R_{x \mid \mathrm{c}^{\prime}}$ are the covariance matrices of the variable $\mathrm{X}$, and $N$ is the channel input signal for given values of $\mathrm{c}$ and $\mathrm{c}^{\prime}$, respectively. To apply the three classes in this study, the covariance matrices of each signal are defined as follows:

$$
\mathrm{c}=\mathrm{c}_{I} \text { and } \mathrm{c}^{\prime}=\mathrm{c}_{\mathrm{D} 1}+\mathrm{c}_{\mathrm{D} 2}
$$

where $c_{I}$ is the covariance matrix of the increasing signal for one of the three classes and $\mathrm{c}_{D 1}, \mathrm{c}_{D 2}$ are the covariance matrices of the decreasing signal for two of the three classes. Using the eigenvectors of the generalized eigenvalue, Eq. (1) can be transformed as follows:

$$
R_{x \mid \mathrm{c}} w=\lambda R_{x \mid \mathrm{c}^{\prime}} w
$$

The eigenvectors in Eq. (3) correspond to a spatial matrix $\mathbf{W}$, and thus, the signals can transform the modified signal $\mathbf{Z}=\mathbf{W} \mathbf{x}$.

This study used $x$ as the acquired EEG signal from the 21channel EEG system and identified the differences among the three classes. Through the spatial matrix W of the CSP it can be seen that the signal for one of the two classes increased and the signal of the third class decreased.

\subsection{Support Vector Machine}

For classification, this study used the support vector machine (SVM) algorithm. The SVM algorithm, which is one of the supervised learning algorithms, sorts data into two or more classes using a support vector [9].

The input feature data are determined after the training. The training data comprise a maximum-margin hyperplane, which divides the space following the positions of the classes. When the training set belongs to a certain class, the decision function is defined as follows:

$$
f(x)=\sum_{i=1}^{N} w_{i} \varphi(x)+b
$$

In Eq. (4), $\varphi$ denotes the predefined functions of $x, w$ is the input vector, and $b$ is the bias. The equation can be used to determine the optimal hyperplane in the binary classification problem as follows:

$$
\operatorname{Max} Q(\alpha)=\sum_{i=1}^{N} \alpha_{i}-\frac{1}{2} \sum_{i=1}^{N} \sum_{j=1}^{N} \alpha_{i} \alpha_{j} y_{i} y_{j} K\left(x_{i} x_{j}\right)
$$

subject to

$$
\operatorname{Max} Q(\alpha)=\sum_{i=1}^{N} \alpha_{i} y_{i}=0 a_{i} \geq 0 i=1, \ldots, N
$$

In Eq. (5), $a_{i}$ is the Lagrange coefficient. In order to determine the object function $Q(\alpha)$ that satisfies the maximum value, the decision function can be defined as follows:

$$
f(x)=\sum_{i=1}^{N} \widehat{\alpha}_{i} y_{i} K\left(x_{i} x_{j}\right)+\widehat{b}
$$




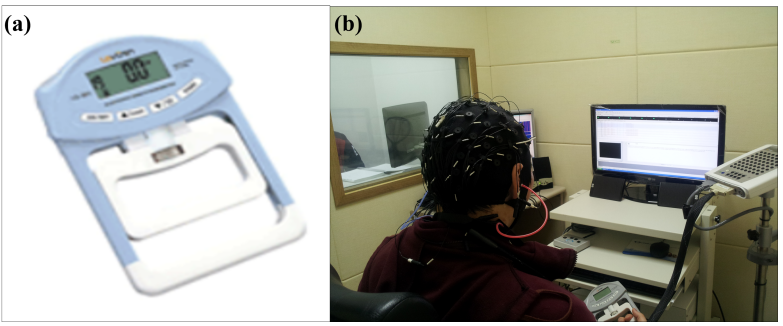

Figure 1. Experiment devices: (a) a digital dynamometer, (b) electroencephalogram equipment.

In Eq. (7), $\widehat{\alpha}_{i}$ and $\widehat{b}$ denote the weighted vectors. This equation classifies the input data. If $f(x) \succ 0$, the data is classified in +1 class; if $f(x) \prec 0$, the data is classified in -1 class.

\section{Experiment}

Five healthy right-handed subjects aged $25-28$ years (four males and one female), without any neurological diseases, participated in the study. The grip strengths of their left and right hands were measured.

\subsection{Digital Dynamometer}

This study measured the grip strength by using a digital dynamometer (Model KS-301; Lavisen Inc., Seoul, Korea) and displayed the results through a digital LCD $(21 \mathrm{~mm})$. The dynamometer is shown in Figure 1(a), which has a maximum gauge of $90 \mathrm{~kg} / 198 \mathrm{lbs}$, a unit of measure of $0.1 \mathrm{~kg} / 0.2 \mathrm{lbs}$, and an allowable error of $0.5 \mathrm{~kg} / 1 \mathrm{lbs}$.

\section{EEG Measurement Device}

To measure the EEG signals of five subjects, this study used a 64-channel EEG system (SynAmps 2; NeuroScan Inc., Charlotte, NC, USA) shown in Figure 1(b). This device can record eye movements using two electrodes placed above and below the left eye. The reference electrodes were placed below both ears and the ground electrode was placed between Fpz and FZ on the forehead. The impedance of each electrode was less than $20 \mathrm{k} \Omega$ and the sampling frequency was $250 \mathrm{~Hz}$.

After EEG data acquisition, this study analyzed the data using the Curry7 software, which is an acquisition and analysis program for the EEG data. Noise due to eye blinking was removed from the measured EEG data and the remaining data were then analyzed with 21-electrode channels. Figure 2 shows the electrodes located in the motor region of the brain $(\mathrm{FCZ}$,

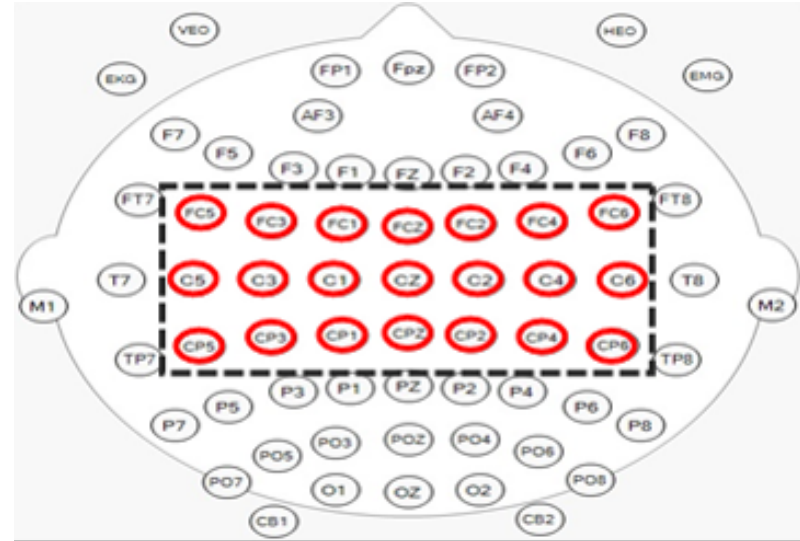

Figure 2. Position of the measurement electrodes.

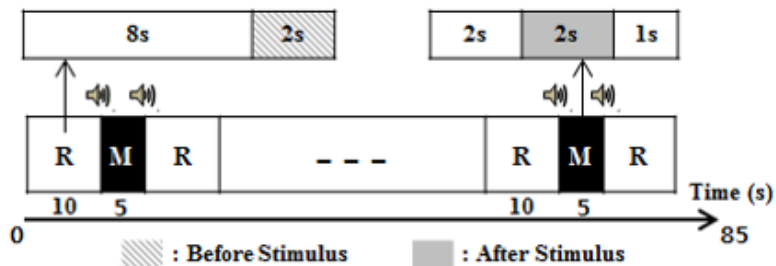

Figure 3. Experimental procedure.

FC1, FC2, FC3, FC4, FC5, FC6, FCZ, C1, C2, C3, C4, C5, C6, $\mathrm{CPZ}, \mathrm{CP} 1, \mathrm{CP} 2, \mathrm{CP} 3, \mathrm{CP} 4, \mathrm{CP}$, and CP6).

\subsection{Experimental Method}

During the experiment, five subjects watched the digital dynamometer LCD displaying their grip strength in the shield room and wore the earphone. Figure 3 shows the experimental procedure. The task was to grip the strength measurement device with their left and right hands. The subjects exerted grip strength at six power levels: $10 \%, 20 \%, 40 \%, 50 \%, 70 \%$, and $80 \%$ of the maximum voluntary contraction (MVC). The subjects maintained voluntary contractions for $5 \mathrm{~s}$. When they heard a beeping sound $(1,500 \mathrm{~Hz}$ for a duration of $200 \mathrm{~ms})$ from the left speaker of earphone, the subjects contracted all their fingers on the dynamometer until they heard a beeping sound (500 Hz and a duration of $200 \mathrm{~ms}$ ) from the right speaker. The rest time was $10 \mathrm{~s}$ between the events.

The total experimental time was $85 \mathrm{~s}$ and data for $2 \mathrm{~s}$ were extracted while maintaining the grip strength for comparison with each event. The extracted data sections were those corresponding to $2 \mathrm{~s}$ before the stimulus (BS) and $2 \mathrm{~s}$ after the stimulus (AS). The subjects performed the task with both hands 


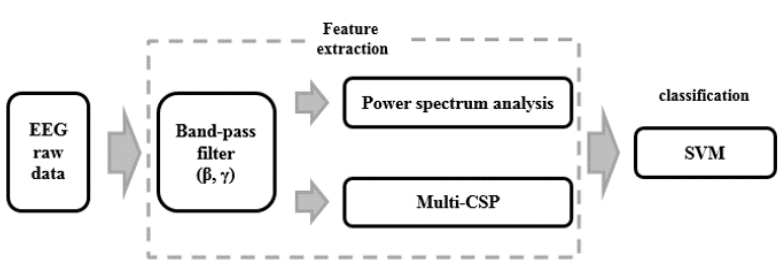

Figure 4. Electroencephalogram (EEG) data process. CSP, common spatial pattern; SVM, support vector machine.

10 times, and the measured grip strength had an error rate of $5 \%$.

\subsection{Feature Extraction Dataset}

This study applied two algorithms, power spectrum analysis and multiclass-CSP, for feature extraction. Figure 4 shows the acquisition process of the feature dataset.

1. Raw EEG data is filtered through the band-pass filter in $\beta$ and $\gamma$ bandwidths.

2. The filtered EEG data for $\beta$ and $\gamma$ bandwidths are extracted through power spectrum analysis and multiclassCSP.

3. SVM classifies the feature datasets into three classes.

In this paper, we acquired EEG data for five subjects at six levels-10\%, $20 \%, 40 \%, 50 \%, 70 \%$, and $80 \%$ of the MVCwhich consisted of three classes: Weak (MVC 10\%-20\%), Medium (40\%-50\%), and Strong (70\%-80\%). Table 1 shows the structure of the feature dataset. From the $\beta$ and $\gamma$ bandwidths of each class, EEG signals were extracted from the feature data through power spectrum analysis and CSP. Onefeature data, which is the mean value of the 21-channel power (AS-BS), were extracted by using power spectrum analysis; two-feature data, which are the maximum and minimum eigenvalues of data obtained from CSP, were extracted at AS by using CSP. The feature dataset consisted of six dimensions. In this study, the SVM algorithm classified the feature dataset into three classes. Five-fold cross-validation was employed as follows:

1. The training set comprised $75 \%$ of the input data and the testing set comprised the remaining data $(25 \%)$.

2. The training set comprised $50 \%$ of the input data and the testing set comprised the remaining data $(50 \%)$.
Table 1. Structure of the feature dataset

\begin{tabular}{cclc}
\hline & Algorithm & Feature data & Dimension \\
\hline $\boldsymbol{\beta}$ & Power spectrum & Mean value & 1 \\
CSP & $\begin{array}{l}\text { Maximum and } \\
\text { minimum value }\end{array}$ & 2 \\
$\gamma$ & Power spectrum & $\begin{array}{l}\text { Mean value } \\
\text { Maximum and } \\
\text { minimum value }\end{array}$ & 1 \\
\hline
\end{tabular}

CSP, common spatial pattern.

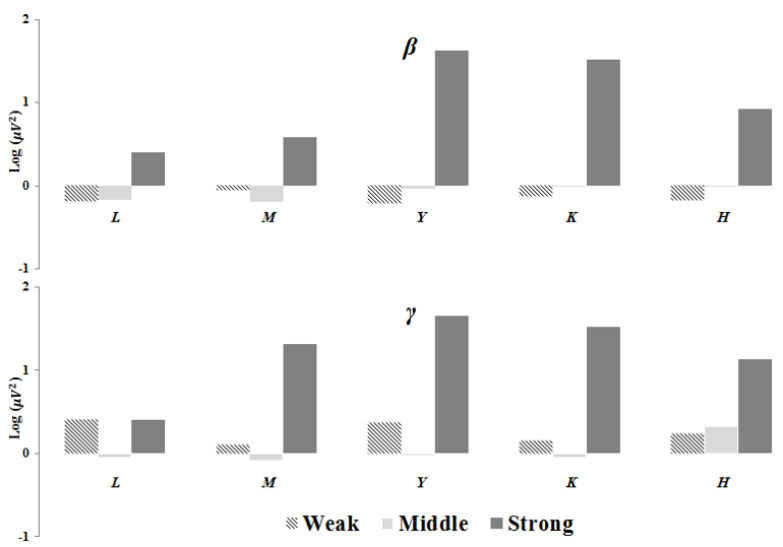

Figure 5. Average of power spectrum analysis for each subject ( $L$, $M, Y, K, H)$ in three classes (Weak, Medium, Strong). Top, $\beta$ $(13-30 \mathrm{~Hz})$; bottom, $\gamma(30-50 \mathrm{~Hz})$.

These two training and test sets were classified using SVM, and the accuracy rate was calculated.

\section{Experimental Results}

\subsection{Power Spectrum Analysis Results}

Figure 5 shows the power spectrum graph. Except for subject $\boldsymbol{M}$, the higher the grip strength, the greater the increase in the EEG power values in $\beta$ waves. Further, in $\gamma$ waves, the power value was the greatest when subjects used strong grip strength However, except for subject $\boldsymbol{H}$, the $\gamma$ power value is smaller for Medium than for Weak, unlike the $\beta$ wave bandwidth.

\subsection{Classification Accuracy Results}

Table 2 shows the results of the accuracy rate of the five-fold cross-validation from the two-test set. Most accuracy rates were highest for the Strong class compared to the other classes. The highest accuracy rate, with an average of $71.3 \%$, was exhibited 
Table 2. Accuracy rate (\%) of each subject

\begin{tabular}{cccc}
\hline & Weak & Medium & Strong \\
\hline $\boldsymbol{L}$ & 53.3 & 42 & 76 \\
$\boldsymbol{M}$ & 30 & 29.3 & 53.3 \\
$\boldsymbol{Y}$ & 58 & 40 & 56.7 \\
$\boldsymbol{K}$ & 6 & 36.7 & 67.3 \\
$\boldsymbol{H}$ & 66.7 & 61.3 & 86 \\
\hline
\end{tabular}

by $\boldsymbol{H}$. With the exception of $\boldsymbol{K}$, the accuracy rate was the lowest for the Medium class as compared to other classes.

\section{Conclusions}

This study investigated the change in EEG signals for $\beta$ and $\gamma$ bandwidths and analyzed the feature datasets with six dimensions using power spectrum analysis and CSP. The feature datasets consisted of three classes and the SVM algorithm was used to classify the datasets. The accuracy rate for most subjects was higher for the Strong class as compared with other classes. Moreover, because of the lower change in the EEG signal for the Weak and Medium classes, the class accuracy rate for these classes was low. In future study, we will try to modify the classification to obtain better accuracy rate for the Weak and Medium classes. If the change in the EEG signal and strength can be precisely classified, or if the EEG-channel associated with the strength can be determined, this study could be applied to many BCI fields.

\section{Conflict of Interest}

No potential conflict of interest relevant to this article was reported.

\section{Acknowledgements}

This work was supported by the Mid-career Researcher Program through an NRF grant funded by the MEST (No. 20120008726).

\section{References}

[1] L. Guo, D. Rivero, J. Dorado, C. R. Munteanu, and A. Pazos, "Automatic feature extraction using genetic programming: an application to epileptic EEG classification," Expert Systems with Applications, vol. 38, no. 8, pp. 10425-10436, 2011. http://dx.doi.org/10.1016/j.eswa. 2011.02 .118

[2] J. D. R. Millan, F. Galan, D. Vanhooydonck, E. Lew, J. Philips, and M. Nuttin, "Asynchronous non-invasive brain-actuated control of an intelligent wheelchair," in Proceedings of International Conference of the IEEE Engineering in Medicine and Biology Society (EMBC2009), Minneapolis, MN, 2009, pp. 3361-3364. http://dx.doi.org/ 10.1109/IEMBS.2009.5332828

[3] D. E. Kim, S. M. Park, and K. B. Sim, "Study on the correlation between grip strength and EEG," Journal of Institute of Control, Robotics and System, vol. 19, no. 9, pp. 853-859, 2013. http://dx.doi.org/10.5302/J.ICROS. 2013.13.1916

[4] D. E. Kim, T. J. Lee, S. M. Park, K. E. Ko, and K. B. Sim, "EEG analysis following change in hand grip force level for BCI based robot arm force control," Journal of Korean Institute of Intelligent Systems, vol. 23, no. 2, pp. 172-177, 2013. http://dx.doi.org/10.5391/JKIIS.2013.23.2.172

[5] D. E. Kim, J. H. Yu, and K. B. Sim, "EEG feature classification for precise motion control of artificial hand,' Journal of Korean Institute of Intelligent Systems, vol. 25, no. 1, pp. 29-34, 2015. http://dx.doi.org/10.5391/JKIIS. 2015.25.1.029

[6] S. I. Lee, "EEG and left/right directional analysis for brain-computer interface," M.S. thesis, Pusan National University, Busan, Korea, 2011.

[7] Y. Tang, J. Tang, and A. Gong, "Multi-class EEG classification for brain computer interface based on CSP," in Proceedings of International Conference on BioMedical Engineering and Informatics (BMEI2008), Sanya, China, 2008, pp. 469-472. http://dx.doi.org/10.1109/BMEI.2008.72

[8] M. Grosse-Wentrup and M. Buss, "Multiclass common spatial patterns and information theoretic feature extraction," IEEE Transactions on Biomedical Engineering, vol. 55, no. 8, pp. 1991-2000, 2008. http://dx.doi.org/10.1109/ TBME.2008.921154

[9] B. E. Boser, I. M. Guyon, and V. N. Vapnik, "A training algorithm for optimal margin classifiers," in Proceedings of the 5th Annual Workshop on Computational Learning 
Theory, Pittsburgh, PA, 1992, pp. 144-152. http://dx.doi. org/10.1145/130385.130401

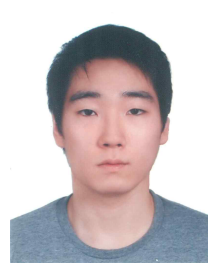

Dong-Eun Kim received his B.S. from the Department of Electrical and Electronics Engineering, Hankyong National University, Anseong, Korea, in 2012 and his M.S. from the Department of Electronic Engineering, Chung-Ang University, Korea, in 2015. His research brain-computer interface, and pattern recognition. Email: jounj@cau.ac.kr

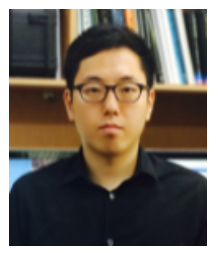

Je-Hun Yu received his B.S. from the Department of Electrical and Electronics Engineering, Chung-Ang University, Seoul, Korea, in 2015. He is currently enrolled in the master's program at the Department of Electrical and Electronics Engineering, Chung-Ang University. His research interests include brain-computer interface, intention recognition, emotion recognition, intelligent robot, intelligence system, internet of things, and big data. E-mail: yjhoon651@ cau.ac.kr

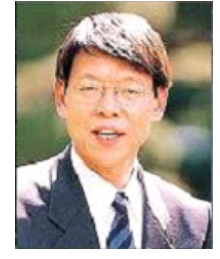

Kwee-Bo Sim received his B.S. and M.S. from the Department of Electronic Engineering, Chung-Ang University, Korea, in 1984 and 1986, respectively. He earned his Ph.D. from the Department of Electrical Engineering, University of Tokyo, Japan, in 1990. Since 1991, he has been a faculty member of the School of Electrical and Electronics Engineering, Chung-Ang University, where he is currently a professor. His research interests include artificial life, intelligent robots, intelligent systems, multi-agent systems, distributed autonomous robotic systems, machine learning, adaptation algorithms, soft computing (neural networks, fuzzy systems, and evolutionary computation), artificial immune systems, evolvable hardware, artificial brain technology, intelligent homes, home networking, intelligent sensors, and ubiquitous computing. He is a member of IEEE, SICE, RSJ, IEICE, KITE, KIEE, and KIIS and is an ICASE Fellow.

Tel: +82-2-820-5319, Fax: +82-2-817-0553

E-mail: kbsim@cau.ac.kr, URL:http://alife.cau.ac.kr 